\title{
Cholinergic modulation during acquisition of olfactory fear conditioning alters learning and stimulus generalization in mice
}

\author{
Eloisa Pavesi, Allison Gooch, Elizabeth Lee, and Max L. Fletcher ${ }^{1}$ \\ Department of Anatomy and Neurobiology, University of Tennessee Health Science Center, Memphis, Tennessee 38163, USA
}

\begin{abstract}
We investigated the role of cholinergic neurotransmission in olfactory fear learning. Mice receiving pairings of odor and foot shock displayed fear to the trained odor the following day. Pretraining injections of the nicotinic antagonist mecamylamine had no effect on subsequent freezing, while the muscarinic antagonist scopolamine significantly reduced freezing. To test whether cholinergic manipulation affected fear generalization, mice were presented with odors similar to the trained odor. Generalization was increased following pretraining scopolamine, while the muscarinic agonist oxotremorine decreased generalization. These results suggest that muscarinic neurotransmission during the acquisition of olfactory association modulates both the strength and specificity of learning.
\end{abstract}

The olfactory system receives cholinergic input from the horizontal limb of the diagonal band of Broca (HDB) (Zaborszky et al. 1986). Blocking olfactory system cholinergic receptors disrupts several aspects of olfactory processing including olfactory shortterm memory (Hunter and Murray 1989; Paolini and McKenzie 1993; Miranda et al. 2009), perceptual learning, and discrimination (Ravel et al. 1992; Fletcher and Wilson 2002; Linster and Cleland 2002; Mandairon et al. 2006). Despite this, relatively little is known about the role of cholinergic activation during acquisition of olfactory aversive learning. While a recent study has demonstrated that cholinergic blockade during acquisition reduces odor-evoked displays of defensive responses to the learned odor (Kroon and Carobrez 2009), the cholinergic contribution to the magnitude and specificity of olfactory fear learning is currently unknown.

Sensory cue-evoked fear conditioning has long been used in the study of the neural mechanisms underlying associative memory. In the standard fear-conditioning paradigm, a neutral stimulus is associated with an aversive foot shock, allowing the conditioned stimulus to acquire an aversive valence and induce fear responses, such as freezing (LeDoux 2003). Olfactory cues have been used as an effective conditioned stimulus in promoting fear-conditioned responses (Otto et al. 2000; Jones et al. 2005; Kroon and Carobrez 2009) and studies in other sensory systems have shown that cholinergic neurotransmission is involved in fear learning (Tinsley et al. 2004). For example, in auditory-cued fear conditioning, blockade of muscarinic receptors disrupts the acquisition of the tone-fear conditioning (Young et al. 1995; Anagnostaras et al. 1999; Feiro and Gould 2005), while blocking nicotinic activation does not affect this learning (Feiro and Gould 2005). Despite this, it remains to be seen whether nicotinic and muscarinic receptors can also mediate conditioned freezing responses to olfactory stimuli.

In this study, we found that pretraining injections of the nicotinic receptor antagonist (nAChR) mecamylamine had no effect on olfactory fear learning. However, pretraining injections of the muscarinic antagonist scopolamine (mAChR) significantly reduced freezing to the trained odor in a dose-dependent manner,

\footnotetext{
${ }^{1}$ Corresponding author

E-mail mfletch4@uthsc.edu

Article is online at http://www.learnmem.org/cgi/doi/10.1101//m.028324.112.
}

suggesting that muscarinic activation is important for the acquisition of olfactory fear learning. We next tested whether manipulation of mAChRs could also affect the specificity of the fear learning. In this case, mice trained to a single odor tend to freeze at similar levels when tested with other structurally similar odors, a phenomenon known as generalization. Overall, we find that generalization is increased following pretraining injections of scopolamine, while pretraining injections of the mAChR agonist oxotremorine decreased the amount of generalization.

Experiments were performed on adult male and female C57BL/6J mice $(n=172)$ and were approved by the University of Tennessee Institutional Animal Care and Use Committee. Mice were group housed with food and water available ad libitum. Methyl scopolamine, scopolamine hydrobromide, mecamylamine, and oxotremorine (Sigma-Aldrich) were dissolved in saline with doses based on previous studies (Castellano and McGaugh 1991; Feiro and Gould 2005). All injections were administered intraperitoneally at $5 \mu \mathrm{L} / \mathrm{g} 10 \mathrm{~min}$ before training. Higher doses of mAChR agonists can cause increased nasal secretions, possibly affecting olfactory-guided behaviors. To avoid this, mice receiving injections of oxotremorine also received the blood-brain barrier impermeant muscarinic antagonist, methyl scopolamine. Previous studies have shown that injections of m-scopolamine have no effect on fear conditioning (Rudy 1996; Anagnostaras et al. 1999). We found that control mice injected with methyl scopolamine alone prior to training displayed freezing levels similar to control levels during testing (M-scop mean freezing $=$ $65.26 \pm 5.7 \%$; Saline mean freezing $=67.84 \pm 5.9 \%$; $t$-test: $t_{(11)}=$ $0.29, P=0.77)$.

All mice were trained inside a mouse test cage equipped with a floor of parallel steel bars connected to a shocker (Coulbourn Instruments). Before training, mice were placed in the chamber for a 10-min habituation period. Conditioning took place in a single session of six trials of a 10-sec ethyl valerate (E5) presentation followed by 0.25 -sec, $0.4 \mathrm{~mA}$ foot shocks. Trials were separated by an ITI of $2 \mathrm{~min}$. Following training, mice were placed back into their home cages until testing the following day.

The next day, mice were placed in a different test chamber with a Plexiglas floor (Med Associates) for a 5-min habituation period. Following habituation, mice received five 20 -sec presentations of the test odorant at 1-min intervals, starting at the second minute. Each session was recorded and analyzed using 
FreezeFrame software (Coulbourn Instruments). Learning was quantified by freezing, defined as the absence of all motor activity in the presence of the cue (Fanselow and Bolles 1979), lasting for at least $2 \mathrm{sec}$ and expressed as the percent time freezing per minute.

Odorants were presented through separate lines into the side of the chambers at $1 \mathrm{~L} / \mathrm{min}$ using an olfactometer. Ethyl butyrate (E4), ethyl valerate (E5), and ethyl hexanoate (E6) (Sigma-Aldrich) were dissolved in mineral oil to achieve an approximate headspace concentration of $200 \mathrm{ppm}$. Statistical significance was determined by one-way ANOVA and post hoc Dunnett's tests using Prism software (GraphPad Software). Error values are reported as mean \pm SEM.

To analyze the effect of $\mathrm{E} 5$ odor in fear-conditioned responses, mice were presented with E5 during the test session. A significant training-condition effect was detected in the percentage of freezing (ANOVA: $\left.F_{(2,18)}=57.98, P<0.05\right)$ with mice in the paired group displaying high levels of freezing $(61.38 \pm 5.4 \%)$ $(n=10)$, while mice in the odor only $(0.00 \pm 0 \%)(n=3)$ and shock only $(3.73 \pm 1.8 \%)(n=7)$ groups froze very little. Post hoc analysis revealed a significant difference in freezing responses between the paired group and the odor only $(P<0.05)$ and the shock only $(P<0.05)$ group (Fig. 1$)$.

To evaluate the role of $\mathrm{m}$ - and nAChRs in the acquisition of conditioned fear, mice received injections of the mAChR antagonist scopolamine $(0.05,0.5$, or $5 \mathrm{mg} / \mathrm{kg})$ or the $\mathrm{nAChR}$ antagonist mecamylamine $(1,2$, or $5 \mathrm{mg} / \mathrm{kg})$ before being placed in the chamber. All groups were trained to E5 and tested with E5 on the following day. For mecamylamine, no statistical differences in freezing times were detected at any dose compared with those receiving sa-
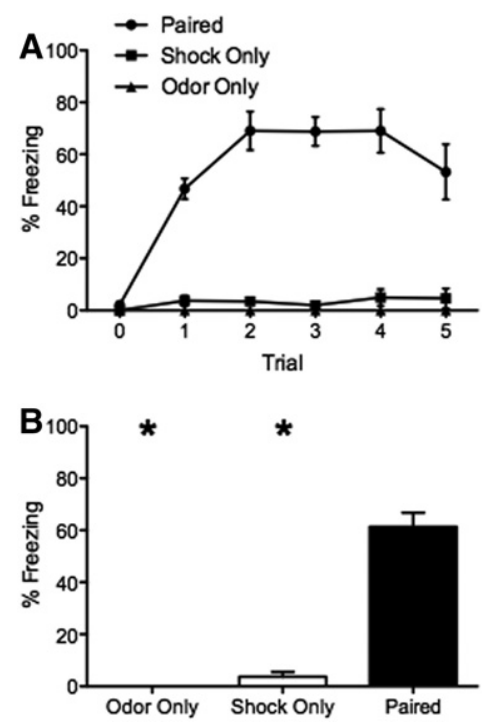

Figure 1. Animals receiving pairings of odor and foot shock showed increased freezing response during a cue test (odor presented in a novel test chamber) $24 \mathrm{~h}$ after training. ( $A$ ) Percent freezing per minute across successive trials on test day. During trial 0 no odor was given to assess baseline freezing levels and potential contextual fear. The trained odor, E5 was presented into the chamber for $20 \mathrm{sec}$ at the beginning of trials $1-5$. Mice were placed into three groups based on training: the paired group $(\bullet)$; the foot shock only group ( $\mathbf{\square})$; and the odor only group ( $\boldsymbol{\Delta})$. From trial 1 on, only the paired group displayed strong freezing responses to E5. (B) Mean time spent freezing across all odor trials for each group. The animals receiving shock only or odor only (white bar) during the training showed little freezing responses during the odor test, whereas those in the paired groups displayed significantly higher freezing levels (black bar). $\left(^{*}\right)$ Significant difference from the control groups, $P<0.05$.
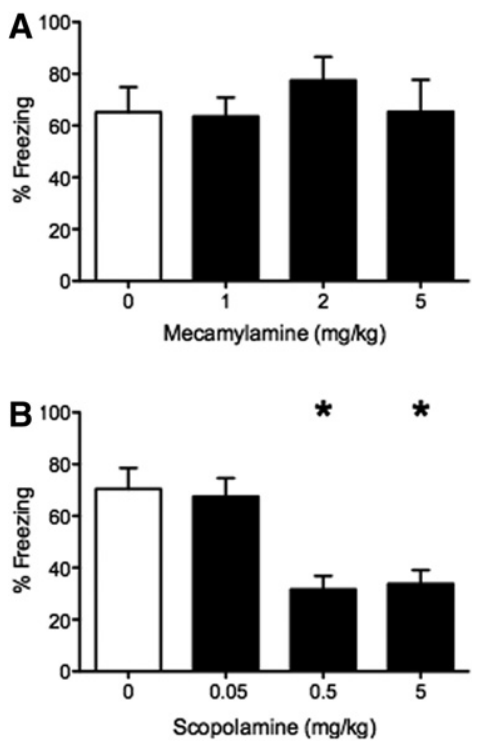

Figure 2. Pretraining muscarinic antagonist but not nicotinic antagonist injections reduce freezing response during a cue test $24 \mathrm{~h}$ after training. $(A)$ Mice received saline (white bar) or mecamylamine (black bars) IP injections $10 \mathrm{~min}$ before the training with odor and foot shock. No differences were found between groups in the freezing responses during the odor test. (B) Mice received saline (white bar) or scopolamine (black bars) injections 10 min before the training with odor and foot shock. The higher doses of scopolamine $(0.5$ and $5 \mathrm{mg} / \mathrm{kg})$ significantly decreased the amount of freezing to the odor during the odor test. $\left(^{*}\right)$ Significant difference from the saline group, $P<0.05$.

line injections (ANOVA: $F_{(3,13)}=0.38, P=0.77$ ) (Fig. 2A). (saline: $65.24 \pm 9.6 \%, n=4 ; 1 \mathrm{mg} / \mathrm{kg}: 63.52 \pm 7.4 \%, n=4 ; 2 \mathrm{mg} / \mathrm{kg}$ : $77.46 \pm 9.1 \%, n=4 ; 5 \mathrm{mg} / \mathrm{kg}: 65.38 \pm 12.3 \%, n=5$ ). For scopolamine, a significant treatment effect was detected between groups in the amount of freezing during testing (ANOVA: $F_{(3,22)}=9.62$, $P<0.05$ ). Pretraining scopolamine injections of 0.5 and $5 \mathrm{mg} / \mathrm{kg}$ decreased the amount of freezing during testing as post hoc analysis revealed significant differences between the saline and $0.5 \mathrm{mg} / \mathrm{kg}(P<0.05)$ and the saline and $5 \mathrm{mg} / \mathrm{kg}$ groups $(P<$ 0.05) (Fig. 2B). (saline: $70.43 \pm 8.1 \%, n=4 ; 0.05 \mathrm{mg} / \mathrm{kg}: 67.54 \pm$ $7.1 \%, n=8 ; 0.5 \mathrm{mg} / \mathrm{kg}: 31.64 \pm 5.2 \%, n=5 ; 5 \mathrm{mg} / \mathrm{kg}: 33.80 \pm$ $5.3 \%, n=9)$.

To determine how learned fear to E5 would generalize to other structurally similar odorants, all mice were trained to E5, but on the following day were placed into three groups. One group was presented with the trained odorant E5; one group with ethyl butyrate (E4), a similar ethyl ester containing one less carbon; and another group with ethyl hexanoate (E6), a similar ethyl ester with one more carbon. Following training, mice presented with E5 during testing displayed similar freezing responses compared with mice in the control experiment $(68.5 \pm 7.5 \%, n=8)$. Mice presented with E4 froze $37.77 \pm 7.1 \%(n=8)$, and mice presented with E6 froze $41.44 \pm 9.5 \%(n=10)$. A significant odor effect was detected between groups during the generalization test (ANOVA: $\left.F_{(2,23)}=3.75, P<0.05\right)$. Post hoc analysis revealed a significant difference in freezing responses between the $\mathrm{E} 4$ group and the E5 group only $(P<0.05)$ (Fig. 3A), suggesting that mice generalize their fear of E5 to the longer chained E6, but not to the shorter chained $\mathrm{E} 4$.

Since blocking mAChRs disrupted learning in our scopolamine study, we next tested whether pretraining scopolamine would also effect fear generalization. The generalization experiment was repeated, with the exception that all mice received 
injections of scopolamine before training. Mice received the $0.5 \mathrm{mg} / \mathrm{kg}$ dose of scopolamine, as it was the lowest dose that significantly reduced freezing in the previous experiment. Mice displayed reduced freezing to E5 $(44.45 \pm 5.4 \%, n=5)$, E4 $(20.28 \pm$ $5.2 \%, n=9)$, and E6 $(28.19 \pm 6.0 \%, n=12)$ (Fig. 3B). No statically significant differences in freezing were observed across odor groups (ANOVA: $F_{(2,23)}=2.98, P=0.07$ ), suggesting that pretraining scopolamine increases generalization.

We next tested whether increased muscarinic activation during learning could decrease generalization of olfactory fear. The experiment was repeated, except that in this case all mice received injections of the mAChR agonist oxotremorine before training to E5. Mice received injections of $0.05 \mathrm{mg} / \mathrm{kg}$ oxotremorine. Mice displayed freezing to E5 similar to saline controls $(66.38 \pm 5.2 \%$, $n=9$; $t$-test: $t_{(15)}=0.23, P=0.82$ ) on the following day. Mice presented with $\mathrm{E} 4$ froze $7.2 \pm 2.0 \%(n=8)$ and mice presented with E6 froze $36.62 \pm 9.9 \%(n=10)$ (Fig. 3C). Overall, a significant effect of the odor was detected by ANOVA $\left(F_{(2,24)}=16.12, P<\right.$ $0.05)$ during the test session. Post hoc tests revealed a significant difference in freezing to E4 and E6 compared with E5, suggesting decreased generalization following increased muscarinic activation during learning.

It is possible that the reduction in learning was due to scopolamine directly disrupting olfactory sensitivity during training. To
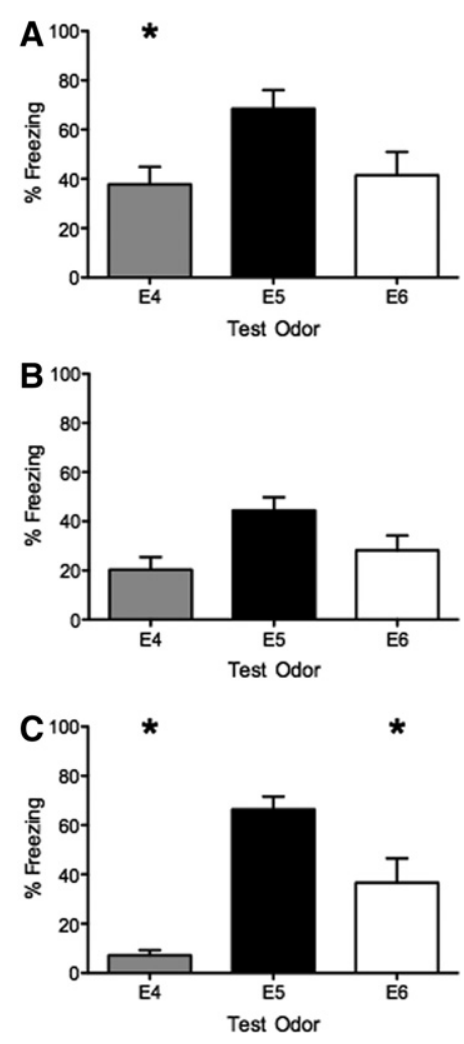

Figure 3. Muscarinic cholinergic activity modulates olfactory fear generalization. All mice were trained with E5 odor and foot shock, and either test with the trained odor E5 or one of the structurally similar odors E4 or E6. (A) In control conditions, mice display significantly less freezing to E4 compared with E5. (B) Pretraining injections of the muscarinic antagonist scopolamine reduced freezing in all test groups, leading to no significant differences in freezing levels in any group, suggesting increased generalization. (C) Pretraining injections of the muscarinic agonist oxotremorine decreased the amount of freezing to both E4 and E6 odors compared with E5, suggesting decreased generalization. (*) Significant difference from the $E 5$ group, $P<0.05$. test whether the animal's ability to detect the odor was diminished with the drugs, we measured olfactory investigation times to $\mathrm{E} 5$ in naive mice following injections of saline, scopolamine $(0.5$ and $5 \mathrm{mg} / \mathrm{kg})$, or oxotremorine + methyl scopolamine $(0.05 \mathrm{mg} / \mathrm{kg})$. Mice were placed in a cage with a $1-\mathrm{cm}$ wooden bead containing filter paper saturated with $0.02 \mathrm{~mL}$ of $\mathrm{E} 5$ dissolved in mineral oil ( $1 \% \mathrm{vol} / \mathrm{vol})$ in the center of the cage. For $1 \mathrm{~min}$, the time each mouse spent investigating a 2-cm-diameter area surrounding the bead was recorded and analyzed using ANY-maze software (Stoelting). We found no significant differences in investigation times across groups, suggesting that the drugs did not significantly impact general olfactory abilities at the doses used here (ANOVA: $F_{(3,28)}=1.72, P=0.19$ ) (saline: $3.45 \pm 0.3 \mathrm{sec}$; scop $0.5 \mathrm{mg} / \mathrm{kg}$ : $2.73 \pm 0.7 \mathrm{sec}$; scop $5 \mathrm{mg} / \mathrm{kg}$ : $2.25 \pm 0.7 \mathrm{sec}$; oxo + m-scop $0.5 \mathrm{mg} / \mathrm{kg}$ : $2.34 \pm 0.3$ ).

Overall, we demonstrated that systemic mAChR, but not nAChR, blockade during acquisition reduces olfactory fear learning in mice. Furthermore, mice trained to associate E5 with foot shock express generalized fear responses toward the longer chained E6, but not toward the shorter chained E4 odor. Pretraining injections of a mAChR antagonist decreased subsequent fear responses to all odors, leading both to a reduction in learning and increase in generalization. Alternatively, pretraining injections of a muscarinic receptor agonist decreased generalization by decreasing freezing responses to both E4 and E6. Overall, these results suggest that $\mathrm{mAChR}$ activation during learning is needed for normal olfactory fear learning to occur.

Our results are consistent with previous studies demonstrating impaired olfactory learning following decreased cholinergic activation using other paradigms (Fletcher and Chen 2010). However, our findings differ from olfactory reward-based studies in which animals with HDB lesions do not display any deficits in olfactory associative learning (Roman et al. 1993; Linster et al. 2001). One explanation for this difference could be due to differences in the two associative learning paradigms used. It is possible that olfactory aversive learning might be more dependent on cholinergic modulation than appetitive learning. For example, a recent study using a similar olfactory aversion paradigm in rats also reported disruptions in olfactory learning following pretraining cholinergic blockade (Kroon and Carobrez 2009). This issue could be addressed in future studies by comparing the effects of HDB lesions on olfactory learning in mice trained in either olfactory reward or aversive conditioning.

Our results are similar to studies reporting reduced auditory fear learning following pretraining $\mathrm{mAChR}$ antagonist injections, but no effects following pretraining nAChR antagonists. Similar to our findings, the same pretraining doses of mecamylamine have no effect on auditory fear learning (Feiro and Gould 2005). Additionally, several studies have reported that auditory fear conditioning is also impaired by pretraining scopolamine treatment (Young et al. 1995; Rudy 1996; Anagnostaras et al. 1999).

Overall, there are several possibilities for the reduction in the learning that has been observed. For example, the blockade of mAChRs may attenuate olfactory sensitivity, alter sensitivity to the foot shock, and/or alter the odor-shock associative plasticity. Our odor investigation experiment along with another report (Doty et al. 2003) suggests that scopolamine does not significantly affect olfactory processing, at least with the odorant concentrations used here. A prior study has also demonstrated that systemic scopolamine does not affect foot-shock sensitivity in mice (Anagnostaras et al. 1999). Thus, the observed impairment in fear conditioning is likely due to disruption of the neural mechanisms involved in olfactory associative learning itself.

In terms of associative conditioning, generalization refers to the tendency of the subject to respond to other stimuli that are 
similar to the learned stimulus. In most cases, the more a stimulus perceptually resembles the learned stimulus, the greater the response (Honig and Urcuioli 1981; McLaren and Mackintosh 2002). By presenting an animal with odorants structurally similar to the trained odor and measuring freezing, we observed how much fear generalization occurred under our training conditions. Compared with E5, mice froze significantly less to E4 while freezing at similar levels to E6, suggesting that mice display significant generalization of their learned fear to E6. Pretraining scopolamine significantly increased this generalization as the difference in freezing between E5 and E4 was reduced following training. Thus, blocking muscarinic activation during learning not only reduces the strength of association, but also reduces the specificity of the learning. These results are consistent with a previous study demonstrating that pretraining scopolamine during olfactory aversive conditioning reduces learning-dependent olfactory acuity the following day (Fletcher and Wilson 2002).

Other studies have also reported that a reduction in cholinergic activation during expression can increase generalization in odor-reward tasks (Linster et al. 2001; Linster and Cleland 2002). However, in these studies, reduced cholinergic activation via HDB lesions was present during both the training and testing phases. Given that these animals displayed no deficits in acquiring the association, it likely that the increased generalization observed is due to the reduction in cholinergic activation during the test itself (Linster and Cleland 2002). This together with the fact that cholinergic activity was only blocked during learning and not during testing in our study suggests that the cholinergic system plays an important role in both olfactory learning and subsequent recognition.

We also tested whether increased muscarinic activation during learning could lead to decreased generalization. Oxotremorine given prior to training significantly decreased freezing to $\mathrm{E} 4$ and E6 compared with E5. These results suggest that muscarinic enhancement during learning may increase the specificity of the learned stimulus. Interestingly, pretraining oxotremorine did not increase the strength of the association, suggesting that memory acuity can, at least in this case, be regulated independent of the strength of the memory. One potential issue with these results lies in the fact that higher doses of oxotremorine have been shown to have analgesic effects in mice (Wess et al. 2003), making them less sensitive to the shock. However, this is likely not an issue here as our dose of oxotremorine was lower than the effective doses for inducing analgesia in the above study.

While these results demonstrate that olfactory generalization can be modulated by mAChRs during olfactory aversive learning, generalization can also be affected by other factors such as CS intensity, US intensity, and training conditions (Baldi et al. 2004; Cleland et al. 2009; Chen et al. 2011). While our paradigm serves as the starting point for investigations into cholinergic influence on olfactory generalization, future experiments manipulating these factors are needed to fully characterize generalization of olfactory fear learning.

Given the global nature of the cholinergic block in this study, it is not possible to identify the affected brain regions that lead to the disruptions in learning reported here. All olfactory-related regions as well as fear-related regions such as the amygdala receive cholinergic input and are sites of interest. Given the numerous reports of cholinergic modulation of olfactory responses (Wilson 2001; Chaudhury et al. 2009; Ma and Luo 2012), it is possible that AChR activation in olfactory areas plays an important role in the modulation of this type of learning. Future studies specifically targeting muscarinic cholinergic receptor activity in the olfactory bulb and piriform cortex during learning will be key in furthering our understanding of the role of acetylcholine in olfactory fear conditioning and generalization.

\section{Acknowledgments}

The work was supported by NIH NIDCD grant DC009853 (M.L.F.) and the Pew Scholars Program (M.L.F.).

\section{References}

Anagnostaras SG, Maren S, Sage JR, Goodrich S, Fanselow MS. 1999. Scopolamine and Pavlovian fear conditioning in rats: Dose-effect analysis. Neuropsychopharmacology 21: 731-744.

Baldi E, Lorenzini CA, Bucherelli C. 2004. Footshock intensity and generalization in contextual and auditory-cued fear conditioning in the rat. Neurobiol Learn Mem 81: 162-166.

Castellano C, McGaugh JL. 1991. Oxotremorine attenuates retrograde amnesia induced by post-training administration of the GABAergic agonists muscimol and baclofen. Behav Neural Biol 56: 25-31.

Chaudhury D, Escanilla O, Linster C. 2009. Bulbar acetylcholine enhances neural and perceptual odor discrimination. J Neurosci 29: $52-60$.

Chen CF, Barnes DC, Wilson DA. 2011. Generalized vs. stimulusspecific learned fear differentially modifies stimulus encoding in primary sensory cortex of awake rats. J Neurophysiol 106: 3136-3144.

Cleland TA, Narla VA, Boudadi K. 2009. Multiple learning parameters differentially regulate olfactory generalization. Behav Neurosci 123: $26-35$.

Doty RL, Bagla R, Misra R, Mueller E, Kerr KL. 2003. No influence of scopolamine hydrobromide on odor detection performance of rats. Chem Senses 28: 761-765.

Fanselow MS, Bolles RC. 1979. Naloxone and shock-elicited freezing in the rat. J Comp Physiol Psychol 93: 736-744.

Feiro O, Gould TJ. 2005. The interactive effects of nicotinic and muscarinic cholinergic receptor inhibition on fear conditioning in young and aged C57BL/ 6 mice. Pharmacol Biochem Behav 80: 251-262.

Fletcher ML, Chen WR. 2010. Neural correlates of olfactory learning: Critical role of centrifugal neuromodulation. Learn Mem 17: 561-570.

Fletcher ML, Wilson DA. 2002. Experience modifies olfactory acuity: Acetylcholine-dependent learning decreases behavioral generalization between similar odorants. J Neurosci 22: 1-5.

Honig WK, Urcuioli PJ. 1981. The legacy of Guttman and Kalish (1956): Twenty-five years of research on stimulus generalization. J Exp Anal Behav 36: 405-445.

Hunter AJ, Murray TK. 1989. Cholinergic mechanisms in a simple test of olfactory learning in the rat. Psychopharmacology 99: 270-275.

Jones SV, Heldt SA, Davis M, Ressler KJ. 2005. Olfactory-mediated fear conditioning in mice: Simultaneous measurements of fear-potentiated startle and freezing. Behav Neurosci 119: 329-335.

Kroon JA, Carobrez AP. 2009. Olfactory fear conditioning paradigm in rats: Effects of midazolam, propranolol or scopolamine. Neurobiol Learn Mem 91: $32-40$.

LeDoux J. 2003. The emotional brain, fear, and the amygdala. Cell Mol Neurobiol 23: 727-738.

Linster C, Cleland TA. 2002. Cholinergic modulation of sensory representations in the olfactory bulb. Neural Net 15: 709-717.

Linster C, Garcia PA, Hasselmo ME, Baxter MG. 2001. Selective loss of cholinergic neurons projecting to the olfactory system increases perceptual generalization between similar, but not dissimilar, odorants. Behav Neurosci 115: 826-833.

Ma M, Luo M. 2012. Optogenetic activation of Basal forebrain cholinergic neurons modulates neuronal excitability and sensory responses in the main olfactory bulb. J Neurosci 32: 10105-10116.

Mandairon N, Ferretti CJ, Stack CM, Rubin DB, Cleland TA, Linster C. 2006. Cholinergic modulation in the olfactory bulb influences spontaneous olfactory discrimination in adult rats. Eur J Neurosci 24: 3234-3244.

McLaren IP, Mackintosh NJ. 2002. Associative learning and elemental representation: II. Generalization and discrimination. Anim Learn Behav 30: 177-200.

Miranda MI, Ortiz-Godina F, Garcia D. 2009. Differential involvement of cholinergic and $\beta$-adrenergic systems during acquisition, consolidation, and retrieval of long-term memory of social and neutral odors. Behav Brain Res 202: 19-25.

Otto T, Cousens G, Herzog C. 2000. Behavioral and neuropsychological foundations of olfactory fear conditioning. Behav Brain Res 110: 119-128.

Paolini AG, McKenzie JS. 1993. Effects of lesions in the horizontal diagonal band nucleus on olfactory habituation in the rat. Neuroscience 57: $717-724$.

Ravel N, Vigouroux M, Elaagouby A, Gervais R. 1992. Scopolamine impairs delayed matching in an olfactory task in rats. Psychopharmacology 109: $439-443$. 
Roman FS, Simonetto I, Soumireu-Mourat B. 1993. Learning and memory of odor-reward association: Selective impairment following horizontal diagonal band lesions. Behav Neurosci 107: 72-81.

Rudy JW. 1996. Scopolamine administered before and after training impairs both contextual and auditory-cue fear conditioning. Neurobiol Learn Mem 65: 73-81.

Tinsley MR, Quinn JJ, Fanselow MS. 2004. The role of muscarinic and nicotinic cholinergic neurotransmission in aversive conditioning: Comparing pavlovian fear conditioning and inhibitory avoidance. Learn Mem 11: 35-42.

Wess J, Duttaroy A, Gomeza J, Zhang W, Yamada M, Felder CC, Bernardini N, Reeh PW. 2003. Muscarinic receptor subtypes mediating central and peripheral antinociception studied with muscarinic receptor knockout mice: A review. Life Sci 72: 2047-2054.
Wilson DA. 2001. Scopolamine enhances generalization between odor representations in rat olfactory cortex. Learn Mem 8: 279-285.

Young SL, Bohenek DL, Fanselow MS. 1995. Scopolamine impairs acquisition and facilitates consolidation of fear conditioning: Differential effects for tone vs context conditioning. Neurobiol Learn Mem 63: $174-180$.

Zaborszky L, Carlsen J, Brashear HR, Heimer L. 1986. Cholinergic and GABAergic afferents to the olfactory bulb in the rat with special emphasis on the projection neurons in the nucleus of the horizontal limb of the diagonal band. J Comp Neurol 243: $488-509$.

Received August 22, 2012; accepted in revised form September 21, 2012. 


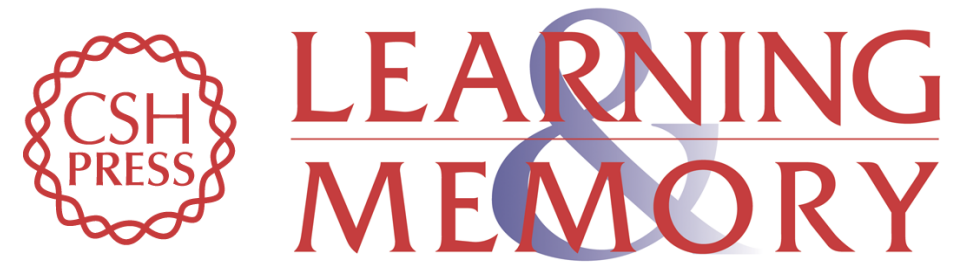

\section{Cholinergic modulation during acquisition of olfactory fear conditioning alters learning and stimulus generalization in mice}

Eloisa Pavesi, Allison Gooch, Elizabeth Lee, et al.

Learn. Mem. 2013, 20:

Access the most recent version at doi:10.1101/Im.028324.112

References This article cites 32 articles, 6 of which can be accessed free at: http://learnmem.cshlp.org/content/20/1/6.full.html\#ref-list-1

License

Email Alerting Receive free email alerts when new articles cite this article - sign up in the box at the Service top right corner of the article or click here. 